\title{
Ignoring Outpatient Excisional Products Can Be Disastrous: Case Presentation
}

\author{
Abdolreza Rouientan ${ }^{1}$, Mostaan Haghpanah Aski ${ }^{1}$ and Hojjat Molaei ${ }^{2 *}$ \\ ${ }^{1}$ Department of Plastic and reconstructive surgery, Shahid Beheshti University of Medical Sciences, Iran \\ ${ }^{2}$ Department of Plastic and reconstructive surgery, University of Medical Sciences, Iran
}

*Corresponding author: Hojjat Molaei, Department of Plastic and reconstructive surgery, Imam Khomeini Hospital Centre, medicine faculty, University of Medical Sciences, Iran

\section{ARTICLE INFO}

Received: 幽 December 13, 2019

Published: 幽January 06, 2020

Citation: Abdolreza Rouientan, Mostaan Haghpanah Aski, Hojjat Molaei. Ignoring Outpatient Excisional Products Can Be Disastrous: Case Presentation. Biomed J Sci \& Tech Res 24(2)-2020. BJSTR. MS.ID.004010.

\section{ABSTRACT}

Background: Scalp neoplasms as one the most common complains, are among routine operations which surgeons and general practitioners are familiar. Obtained products are significant materials to deal. During recent era multiple challenges evoked about cost - benefit tissue examination and insurances presented guidelines to illustrate their main rout.

Methods and Materials: Here, there is a complicated case of simple head lump, which would be an easy to solve problem, but unfortunately improper decision in sending tissue examination resulted in a catastrophe of cosmetic and functional sequels and she tolerated multiple procedures to reach acceptable result.

Results and Conclusion: The presented patient underwent multiple operations to reconstruct resulted defect. Despite challenges on decision about histologic examination of skin specimen, it seems that a simple tissue diagnosis can be assume lifeboat of unfortunate patients.

\section{Background}

Scalp neoplasms are common finding among western populations [1] and mostly are cysts in forms of epidermoid, dermoid and trichilemmals according to their contents [2]. Dermoid cysts contain ectoderm and skin elements, whereas in epidermoid cysts, cutaneous elements are eliminated but ectoderm $[3,4]$. These sequestrations of tissues in fetal development are basics in pathogenesis. However, retained previous surgical events can mimic this process [4]. There are generally accepted agreements about fate of excised lesions by medical practitioners, which highlighted by Brazilian Society of Pathology as: "the pathological examination of specimens or parts removed from a human body should be performed by physicians acting with utmost care and using the best of their professional expertise, always to benefit patients and never as an obligation." and "the patient is the owner of his/her cells, tissues and organs, which cannot be simply discarded at the discretion of physicians. It is necessary to explain why tissues are excised, as well as to inform the objectives of a pathological procedure, and the patient consents or not about discarding his/ her [remaining] material [5]." Jeyakumar et al. [6] support tissue examination of excised cysts as follows: they recommend the specimen be transected peri-operatively to further support the diagnosis. If the contents are cheesy material consistent with an epidermoid cyst, then the diagnosis is confirmed, and the specimen can be discarded. If there is diagnostic ambiguity, or the contents are solid, the specimen should be sent for histological examination [6]. As Klebanov et al. [7] highlight misdiagnosis of malignant masses of scalp such as pleomorphic dermal sarcoma of the scalp [7]. Here, we present an ignored case which discarded primary cutaneous cyst resulted in complicated events and patient had to tolerate complex procedures and eventually discuss about possible recommendations.

\section{Case Presentation}

The patient, a 37-year-old woman referred us complaining for a very large scalp mass developed since the previous four 
months Figure 1. She had a history of two operations because of scalp lesion. The first time was eight months ago when a general physician excised one $\mathrm{cm}$ scalp lesion and discarded the specimen without referral to tissue examination, and due to relapse of the lesion, another surgery about four months later for an eight-cm lesion by clinical impression of the relapse of dermoid cyst, which was excised by a general surgeon. The pathology report was a dermoid cyst. There was no other past medical history or familial history of similar lesion. When the patient referred to our ward, she had a very large scalp mass that involved partially the entire scalp. In examination, the mass was firm, and it had adhesions due to the scars from the past twice surgeries in central part. MRI evaluation was made and a large soft tissue mass with some parts of cyst formation due to necrosis were reported; no invasion to scalp bone was reported Figure 2.

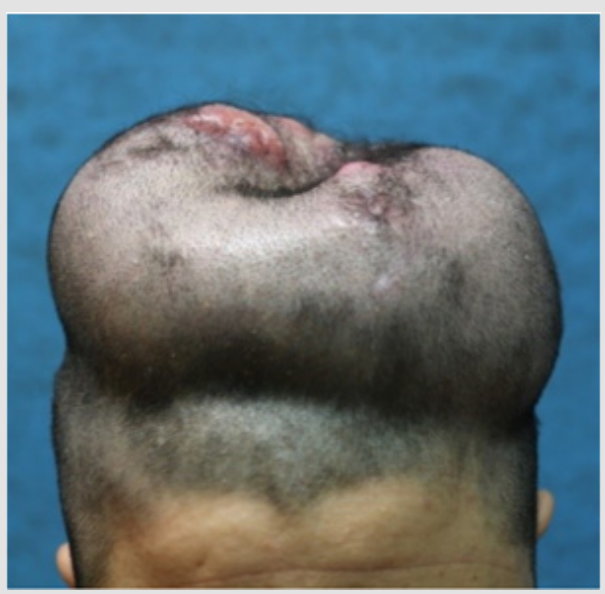

Figure 1: 38 years old female admitted for a big scalp lesion.

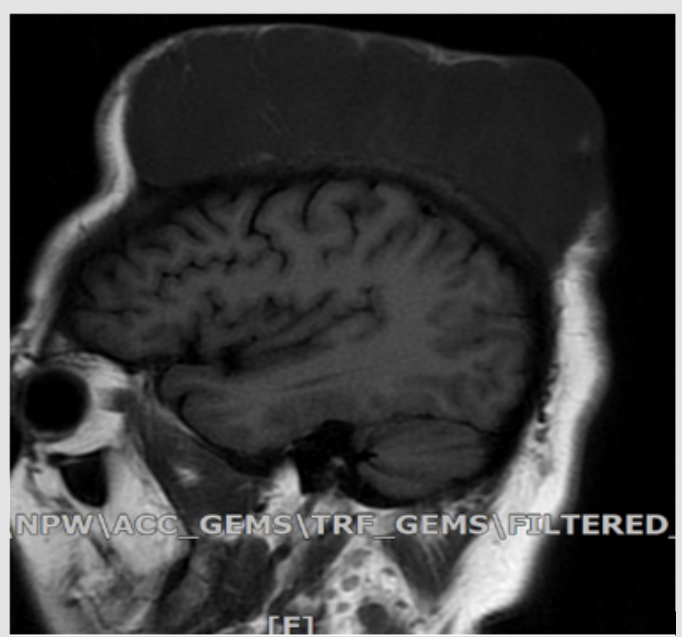

Figure 2: Sagittal MRI demonstrating large soft tissue mass with some parts of cyst formation due to necrosis before radical excision and reconstruction.

After evaluation, the patient was ready for the operation. First, using an elliptical incision, excision of the mass was made, and the sample was sent for frozen section evaluation, which reported malignant tumor in favor of origin from dermatofibrosarcoma protuberans with bone involvement and margins free of tumor. Therefore, re-excision with $6-\mathrm{cm}$ margin was made and due to central vertex bone involvement, bone excision of about $8 \mathrm{~cm}$ diameter of external table was done and the scalp defect was covered with partial thickness skin graft from thigh Figure 3 . Because of doubt in the previous pathologic report, reevaluation was made and dermatofibrosarcoma protuberans was reported for the previous sample Figure 4. The final pathology report was spindle cell sarcoma compatible with fibrosarcoma arising on dermatofibrosarcoma protuberans with $18^{*} 17^{*} 5 \mathrm{~cm}$ diameter and margins free of tumor. After one month follow up, no evidence of tumor relapse was observed in examination and MRI and the patient was referred for radiotherapist consultation. Three months after the operation, the patient was fine, and no recurrence was seen Figure 5. It is very important that every excision sample from body be sent for pathology. In the case we reported in the present study, we can see how a simple problem may result in serious complications if ignored.

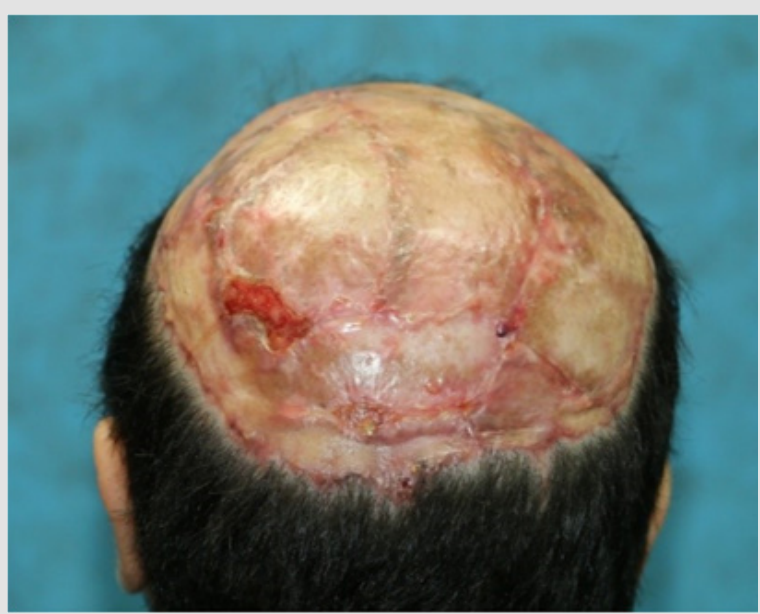

Figure 3: The same patient a few months later without recurrence.

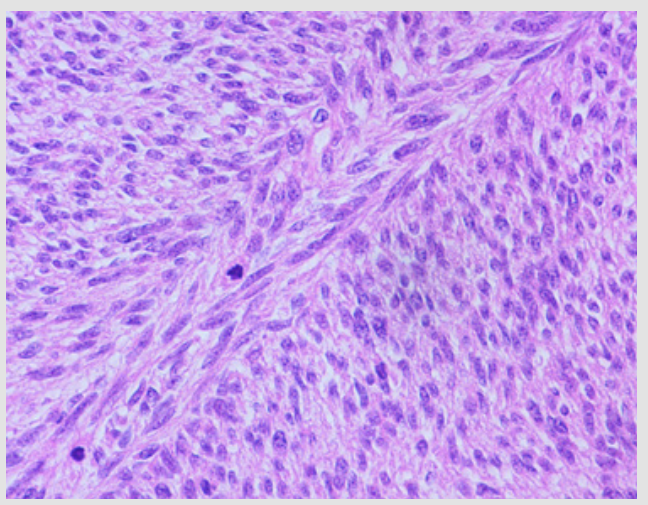

Figure 4: Sections show a hyper cellular malignant neoplastic growth composed of interlacing long spindle cells having hyper chromatic mitotically active nuclei (14/10 HPF) forming herring bone appearance in most parts; necrosis is not seen. 


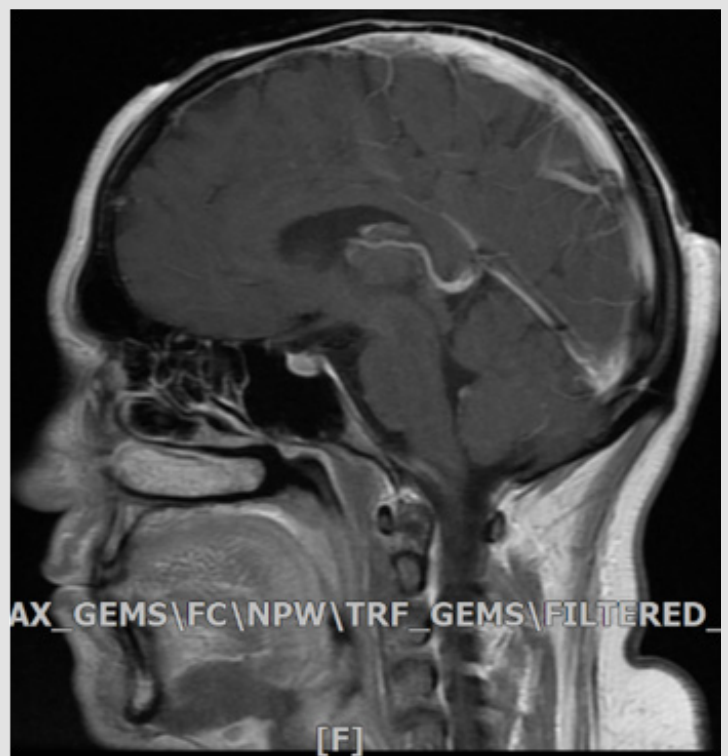

Figure 5: Sagittal MRI demonstrating eradicated lesion without recurrence a few months after surgery.

\section{Discussion}

Wide extended area of the scalp contains various anatomical structures which can include broad range of tumor types that may arise in this region, including over a hundred different neoplasms, hamartomas, malformations and cysts, both benign and malignant [2]. Leung reported a case of trichilemmal cyst which was misdiagnosed as a simple cyst and highlighted differential diagnoses of scalp lump including epidermoid cysts, benign lipomas, spindle cell lipoma, hibernomas and liposcarcoma [8]. Our patient had similar scenario and had to pass unwanted operations with longer recovery periods. It is estimated head and neck primary sarcomas represent approximately 5\%-15\% of all adult sarcomas. Despite rarity, fibrosarcoma is one of the more common types of soft-tissue sarcoma. In developed imaging like CT, fibrosarcoma looks like as a homogeneous appearance with variable enhancement and bone remodeling. It has low to intermediate signal intensity with all MR imaging sequences. CT and MR imaging both demonstrate extension or infiltration into adjacent structures [9].

Dermatofibrosarcoma protuberans is a superficial, low-grade, locally aggressive neoplasm in trunk and proximal extremities with high recurrence rate. It begins as a dermal plaque or nodule from fibroblasts and grows slowly, sometimes becoming multinodular confused with multiple lesions. Though, composed of uniform spindle tumor cells with minimal cytoplasm and an indistinct margin [10]. In the head region in particular, there is a high rate of local recurrences, even if established sarcoma was initially excised with wide $(2-3 \mathrm{~cm})$ surgical margin and Rapid growth and potential tissue necrosis can make it difficult to distinguish such a lesion from an infected benign trichilemmal cyst. Moreover, low accurate preoperative diagnosis warrants referral suspicious lesions to histological confirmation [2].
Improperly educated surgeons who have incomplete clinical judgment, need histological examination to confirm their diagnosis of simple cysts, though, this increases insurance demands on health system. So, Jeyakumar et al. [6] recommended red flags of diagnosis in simple lumps [6]. On the other hand, there is not always complete correlation between biopsy and final tissue examination, as mentioned by Han et al in a retrospective study on results of tissue examination reports of 1867 non-melanoma skin cancer specimen [11]. We had similar problem between $2^{\text {nd }}$ and $3^{\text {rd }}$ surgery tissue examination, even between frozen section examination and final tissue evaluation that seems to be related to nature of cutaneous spindle sarcomas. Kirby et al. [12] suggested histological examination of tissue removed by GP's always necessary because routine histological examination might detect cutaneous tumors [12]. In a comprehensive evaluation of patient's scenario, we recommend every general practitioner not to discard excised simple cysts and send to tissue examination, though, it may have tough opponents to discuss for cost benefit decisions.

\section{Acknowledgment}

Authors thank Dr. Alireza Abdollahi AP, CP, MD for preparing valuable histologic figures.

\section{Conflict of Interests}

None of the authors declare any conflict of interest.

\section{References}

1. Al khateeb TH, Al Masri NM, Al Zoubi F (2009) Cutaneous cysts of the head and neck. J Oral Maxillofac Surg 67(1): 52-57.

2. Prodinger CM, Koller J, Laimer M (2018) Scalp tumors. J Dtsch Dermatol Ges 16(6): 730-753.

3. Ghassan A Tranesh, Hong Qu (2019) Skin nonmelanocytic tumors Cysts Bronchogenic cyst (cutaneous). PathologyOutlines.com.

4. Sabhalok SS, Shetty LS, Sarve PH, Setiya SV, Bharadwaj SR (2016) Epidermoid and dermoid cysts of the head and neck region. Plast Aesthet Res 3: 347-350.

5. Werner B (2009) Skin biopsy and its histopathologic analysis: Why? What for? How? Part I. An Bras Dermatol 84(4): 391-395.

6. Jeyakumar R Apollos, Gregory E Ekatah, Guat Shi Ng, Angus K McFadyen, Stuart C Whitelaw (2017) Routine histological examination of epidermoid cysts; to send or not to send? Annals of Medicine and Surgery 13: 24-28.

7. Klebanov N, Hoang MP, Reddy BY (2018) Pleomorphic Dermal Sarcoma of the Scalp. Cureus 10(7): e2979.

8. Leung LK (2011) Differential diagnosis of soft scalp lumps. BMJ Case Rep.

9. Morón FE, Morriss MC, Jones JJ, Hunter JV (2004) Lumps and bumps on the head in children: use of CT and MR imaging in solving the clinical diagnostic dilemma. Radiographics 24(6): 1655-1674.

10. Choi JH, Ro JY (2018) Cutaneous Spindle Cell Neoplasms Pattern-Based Diagnostic Approach Arch Pathol Lab Med 142(8): 958-972.

11. Han J, Nosrati NN, Soleimani T, Munshi IA, Flores RL, et al. (2016) Analysis of Cases in Which a Biopsy Specimen Is Positive and an Excised Lesion Is Negative for Nonmelanoma Skin Cancer. JAMA Surg 151(5): 486-488. 
12. Kirby B, Harrison P, Blewitt R (1998) Is histological examination of tissue removed by GP's always necessary. More skin carcinomas might be detected with routine histological examination. BMJ 316(7133): 779.

\section{ISSN: 2574-1241}

DOI: 10.26717/BJSTR.2020.24.004010

Hojjat Molaei. Biomed J Sci \& Tech Res

(C) (i) This work is licensed under Creative

Submission Link: https://biomedres.us/submit-manuscript.php

$\begin{array}{ll}\text { BIOMEDICAL } & \text { Assets of Publishing with us } \\ \text { RESEARCHES } & \text { - Global archiving of articles } \\ & \text { - Immediate, unrestricted online access } \\ & \text { - Rigorous Peer Review Process } \\ \end{array}$

